\title{
Rotranslational state-to-state rates and spectral representation of inelastic collisions in low-temperature molecular hydrogen
}

\author{
S. Montero ${ }^{\mathrm{a})}$ \\ Instituto de Estructura de la Materia, CSIC, Serrano 121, 28006 Madrid, Spain \\ F. Thibault \\ Laboratoire de Physique des Atomes, Lasers, Molécules et Surfaces, UMR-CNRS 6627, \\ Université de Rennes I, Campus de Beaulieu, F-35042 Rennes Cedex, France
}

G. Tejeda and J. M. Fernández

Instituto de Estructura de la Materia, CSIC, Serrano 121, 28006 Madrid, Spain

(Received 10 July 2006; accepted 16 August 2006; published online 22 September 2006)

\begin{abstract}
Inelastic collisions in natural $\mathrm{H}_{2}$ are studied from the experimental and theoretical points of view between 10 and $140 \mathrm{~K}$. Rotational populations and number densities measured by Raman spectroscopy along supersonic expansions of $\mathrm{H}_{2}$ provide the link between experimental and theoretical rotranslational state-to-state rate coefficients of $\mathrm{H}_{2}$ in the vibrational ground state. These rates are calculated in the close- scattering approach with the MOLSCAT code employing a recent $a b$ initio $\mathrm{H}_{2}-\mathrm{H}_{2}$ potential. The calculated rates are assessed by means of a master equation describing the time evolution of the experimental rotational populations. The feasibility for obtaining the rates on the sole basis of the experiment is discussed. The dominant processes $j_{1} j_{2} \rightarrow j_{1}^{\prime} j_{2}^{\prime}$ in the investigated thermal range are found to be $21 \rightarrow 01>30 \rightarrow 12>31 \rightarrow 11$, proving the importance of double processes such as $30 \rightarrow 12$. Good agreement is found between theory and experiment, as well as with earlier ultrasonic measurements of relaxation times. A spectral representation is proposed in order to visualize quantitatively the collisional contributions in any nonequilibrium time evolving process. () 2006 American Institute of Physics.
\end{abstract}

[DOI: $10.1063 / 1.2353121]$

\section{INTRODUCTION}

Much of the ongoing research on molecular collisions is aimed at establishing accurate state-to-state collisional cross sections and their associated rate coefficients for a number of small molecular species in a wide range of temperatures. Such data are fundamental for a detailed understanding of energy transfer in elementary collisional processes.

State-to-state cross sections and rates derived from quantum calculations are highly sensitive to the intermolecular potential energy surface (PES) employed in the calculation. ${ }^{1}$ Accurate experimental cross sections or rates therefore allow one to assess the accuracy of different PESs. However, the experimental methods developed so far only yield indirect information about state-to-state quantities. Therefore, testing the quality of a PES by comparison with experimental stateto-state quantities is indeed a difficult problem whose solution demands a close cooperation of theoreticians and experimentalists. Perhaps the paradigm of this problem is molecular hydrogen, with much theoretical ${ }^{1-9}$ and experimental material ${ }^{10-18}$ available, which is not of immediate interpretation in most cases. Here we tackle the problem from both sides, experiment and theory, exploring novel experimental techniques with the support of close-coupling scattering calculations ${ }^{19}$ based on a recent ab initio PES for $\mathrm{H}_{2}-\mathrm{H}_{2}{ }^{20}$

\footnotetext{
a)Electronic mail: emsalvador@iem.cfmac.csic.es
}

Among the environments which depend on nonreactive collisions with $\mathrm{H}_{2}$ molecules, the interstellar space and the molecular clouds have attracted much attention in recent times. Modeling such rarefied gas media, which are under severe nonequilibrium conditions, is a central problem of present day molecular astrophysics. ${ }^{21-26}$ Other topics such as teledetection based on collisional line broadening, ${ }^{10-12}$ quantification of molecular transport properties, ${ }^{2}$ and relaxation phenomena are still open problems in spite of the progress in kinetic theory and computation power during the last three decades.

The range $5 \leqslant T \leqslant 300 \mathrm{~K}$ investigated here for molecular hydrogen spans the lower end of the thermal domain of astrophysical interest.

\section{METHODOLOGY}

The experimental part of this work relies on the master equation $^{4}$ (MEQ)

$$
\frac{d P_{i}}{d t}=n \sum_{j, \ell \leqslant m} Q_{i j \ell m}\left(-P_{i} P_{j} k_{i j \rightarrow \ell m}+P_{\ell} P_{m} k_{\ell m \rightarrow i j}\right)
$$

which describes the time evolution of the population $P_{i}$ of a rotational level with quantum number $J=i$ as a consequence of inelastic collisions in a gas of diatomic molecules at instantaneous number density $n$ and translational or kinetic temperature $T_{t}$. The factor 


$$
\begin{aligned}
Q_{i j \ell m}= & {\left[1+\delta_{i j}\left(1-\delta_{\ell i}\right)\left(1-\delta_{m i}\right)\right] } \\
& \times\left[1-\delta_{\ell i}\left(1-\delta_{i j}\right)\right]\left[1-\delta_{m i}\left(1-\delta_{i j}\right)\right]
\end{aligned}
$$

accounts for the symmetry effects in collisions between indistinguishable molecules. ${ }^{4}$ Indistinguishable molecules will be considered for $\mathrm{pH}_{2}: \mathrm{pH}_{2}$ and $\mathrm{oH}_{2}: \mathrm{oH}_{2}$ collisions, and distinguishable molecules for $\mathrm{oH}_{2}: \mathrm{pH}_{2}$ collisions.

The $k$ 's in MEQ (1) are the so called state-to-state collisional rates (also excitation and deexcitation rates), quantities which account for the elementary collisional process,

$$
\mathrm{H}_{2}(i)+\mathrm{H}_{2}(j) \stackrel{k_{i j \rightarrow \ell m}}{\longrightarrow} \mathrm{H}_{2}(\ell)+\mathrm{H}_{2}(m),
$$

between a $\mathrm{H}_{2}$ molecule in the rotational state $J=i$ and another $\mathrm{H}_{2}$ molecule in the rotational state $J=j$, in a medium at translational temperature $T_{t}$. As a consequence of the collision, the final states of the two molecules become $J=\ell$ and $J=m$.

The collisional rates obey the detailed balance

$$
k_{\ell m \rightarrow i j}=k_{i j \rightarrow \ell m} \frac{(2 i+1)(2 j+1)}{(2 \ell+1)(2 m+1)} e^{\left(E_{\ell}+E_{m}-E_{i}-E_{j}\right) / k_{B} T_{t}},
$$

where $E_{i}, E_{j}, E_{\ell}$, and $E_{m}$ are the energies of the rotational levels involved. For $E_{\ell}+E_{m}>E_{i}+E_{j}$ the upward ("up") and downward ("down") rates will henceforth be labeled as $k_{i j \rightarrow \ell m}^{\text {up }}$ and $k_{\ell m \rightarrow i j}^{\text {down }}$, respectively.

The medium considered here is the gas flowing along the axis of a supersonic expansion with space-time dependent quantities $n(z, t), P_{i}(z, t)$, and $T_{t}(z, t)$. The particular case studied is natural molecular hydrogen $\left(\mathrm{nH}_{2}\right)$, a 3:1 mixture of orthohydrogen $\left(\mathrm{oH}_{2}\right)$ and parahydrogen $\left(\mathrm{pH}_{2}\right)$ with $J$ $=$ odd and $J=$ even, respectively. These variants of $\mathrm{H}_{2}$ do not interconvert in the time scale of the supersonic expansion. The rotational populations in Eq. (1) are then normalized as

$$
\sum_{i=\text { odd }} P_{i}=3 / 4, \quad \sum_{i=\text { even }} P_{i}=1 / 4 .
$$

Since up and down rate coefficients at temperature $T_{t}$ may differ by many orders of magnitude, it is convenient to reformulate the MEQ (1) in terms of only down rate coefficients, which show a far smoother dependence on $T_{t}$. With aid of Eq. (4), the MEQ (1) is then formally rewritten as

$$
d P_{i} / d t=\sum_{\tau \omega \sigma \rho} \pm a_{\tau \omega \sigma \rho} k_{\tau \omega \rightarrow \sigma \rho}^{\text {down }},
$$

with explicit form and $a_{\tau \omega \sigma \rho}$ coefficients defined in the Appendix in terms of the instantaneous experimental quantities $n, P_{i}$, and $T_{t}$. The indices $\tau, \omega, \sigma$, and $\rho$ run over the values of the rotational quantum number $J$ allowed by ortho-para nonconversion, with at least one of the indices being equal to $i$. Only $i=J=0,1$ levels and $\Delta J=\sigma-\tau=0, \pm 2$ and $\Delta J=\rho$ $-\omega=0, \pm 2$ processes are relevant for $\mathrm{nH}_{2}$ at $T_{t} \leqslant 300 \mathrm{~K}$. Each $a_{\tau \omega \sigma \rho} k_{\tau \omega \rightarrow \sigma \rho}$ term in Eq. (6) accounts in a factorized form (instantaneous local properties $\times$ intermolecular properties) for the net contribution of the $\sigma \rho \rightarrow \tau \omega$ and $\tau \omega \rightarrow \sigma \rho$ time-symmetric processes to the population rate $d P_{i} / d t$.

The rotational population rates $d P_{i} / d t$ as well as the $a_{\tau \omega \sigma \rho}$ coefficients are determined directly from the experiment as discussed below. In turn, the $k_{\tau \omega \rightarrow \sigma \rho}$ 's may be obtained from first principle calculations or, with some limitations, from the experiment as shown in Sec. V.

\section{EXPERIMENT}

The experimental quantities involved in the $a_{\tau \omega \sigma \rho}$ coefficients of Eq. (6) and in its left-hand term $d P_{i} / d t$ were determined from the intensities of the $Q(0), Q(1), Q(2)$, and $Q(3)$ Raman lines of $\mathrm{H}_{2}$ at 4161.2, 4155.3, 4143.5, and $4125.9 \mathrm{~cm}^{-1}$, respectively. These intensities were measured at a number of points located along the axis of supersonic expansions of $\mathrm{nH}_{2}$, at distances $z$ from the nozzle orifice. In this region (zone of silence) the flow is laminar, and the distance $z$ can be related with the expansion time $t$ by means of the flow velocity $V(z)=d z / d t$. The population rates are then determined from

$$
\frac{d P_{i}}{d t}=\frac{d P_{i}}{d z} V(z) .
$$

The quantities $P_{i}, d P_{i} / d z$, and $n$ needed in Eq. (6) were measured as described in earlier works, ${ }^{18,27}$ while $V(z)$ was determined assuming the conservation of enthalpy along the expansion axis.

The translational temperature $T_{t}$ at each position $z$ of the expansion axis has been deduced in terms of the corresponding local number density and rotational populations by means of

$$
T_{t}(z)=T_{0}\left[\left(\frac{n(z)}{n_{0}}\right) C \prod_{J}\left(\frac{P_{J}(z)}{g_{J}(2 J+1)}\right)^{P_{J}(z)}\right]^{2 / 3},
$$

with

$$
C=\prod_{J}\left(\frac{P_{J}^{0}}{g_{J}(2 J+1)}\right)^{-P_{J}^{0}},
$$

where $g_{J}=1$ for $J=$ even and $g_{J}=3$ for $J=$ odd are the nuclear spin weights of $\mathrm{pH}_{2}$ and $\mathrm{oH}_{2}$, respectively; $n_{0}$ is the stagnation number density, and $P_{J}^{0}$ are the rotational populations at the stagnation temperature $T_{0}$. Equation (8) has been derived from the statistical definition of entropy, assuming entropy invariance along the axis of the supersonic expansion.

Two independent data sets were measured from two jets of $99.9999 \%$ pure $\mathrm{nH}_{2}$ generated at stagnation pressures $p_{0}$ $=1.28$ and 2.55 bars at $T_{0}=295 \mathrm{~K}$, expanding the gas through a circular nozzle of diameter $D=313 \mu \mathrm{m}$. The remaining experimental conditions are similar to those of Ref. 18. The experimental quantities $n(z), V(z)$, and $P_{i}(z)$ are shown in Figs. 1 and 2. The rotational temperatures of $\mathrm{oH}_{2}$ and $\mathrm{pH}_{2}$ have been measured from the ratio of populations $P_{3} / P_{1}$ and $P_{2} / P_{0}$, with the constraints of Eq. (A1) (Appendix). The rotational and translational temperatures are shown in Fig. 3. The translational temperatures resulting from Eq. (8) are very similar for both expansions. On the contrary, the rotational temperatures differ by up to $\approx 15 \mathrm{~K}$, always with $T_{r}\left(\mathrm{pH}_{2}\right)<T_{r}\left(\mathrm{oH}_{2}\right)$. 


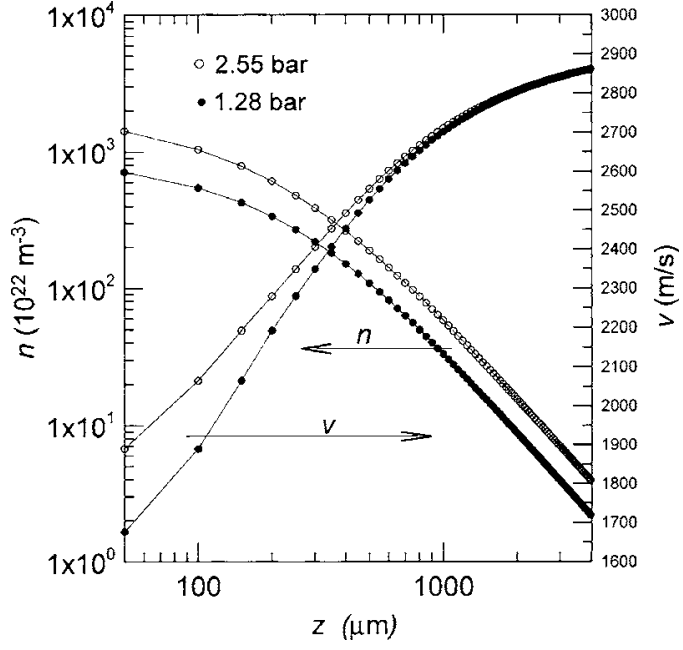

FIG. 1. Number density and flow velocity along the axis of two supersonic expansions of $\mathrm{nH}_{2}$ at stagnation pressures of 1.28 and 2.55 bars, temperature $T_{0}=296 \mathrm{~K}$, and nozzle orifice $D=313 \mu \mathrm{m}$. The lines are a guide to the eye.

The population rates $d P_{i} / d t$ were determined by means of Eq. (7), employing the flow velocities of Fig. 1. The slopes $d P_{i} / d z$ were obtained numerically from the populations $P_{i}(z)$ of Fig. 2. For optimal accuracy, smoothing functions of the form

$$
P_{i}(z)=a_{i}\left(z+z_{0}\right)+b_{i}+c_{i}\left(z+z_{0}\right)^{-2}
$$

were fitted to consecutive seven-point sets. The coefficients $a_{i}, b_{i}$, and $c_{i}$ are different for each set while $z_{0}$ accounts for the true origin of the expansion, the so called sonic surface, where the Mach number reaches the value $M=1$. In the present expansions it appears to be located at $\approx 100$ and $\approx 200 \mu \mathrm{m}$ inside the nozzle for the expansions at 1.28 and 2.55 bars, respectively. The experimental population rates $d P_{0} / d t$ and $d P_{1} / d t$ for the ground rotational states of $\mathrm{pH}_{2}$

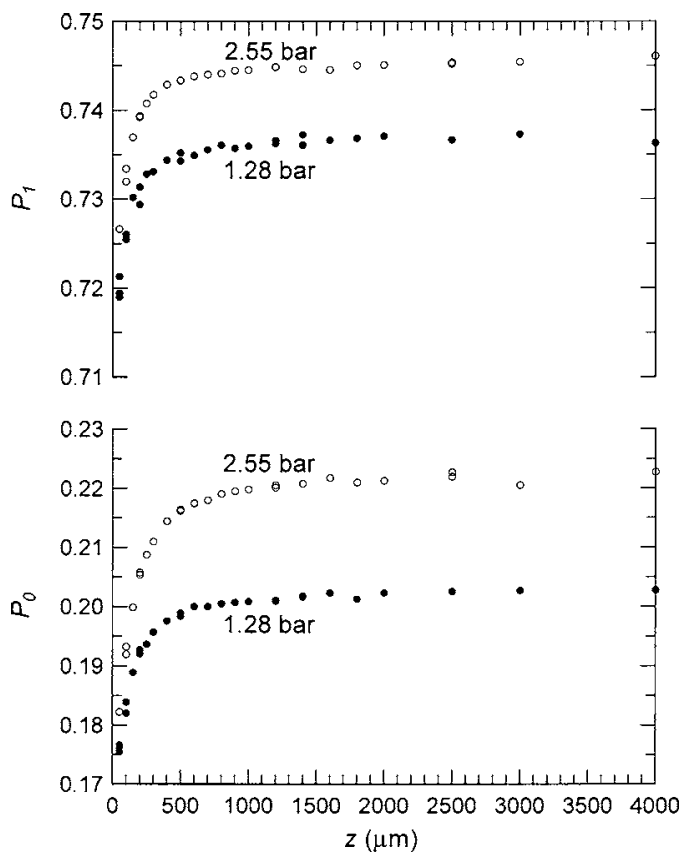

FIG. 2. Population of the rotational levels $J=0$ and $J=1$ along the axis of two supersonic expansions of $\mathrm{nH}_{2}$. Same conditions as in Fig. 1.

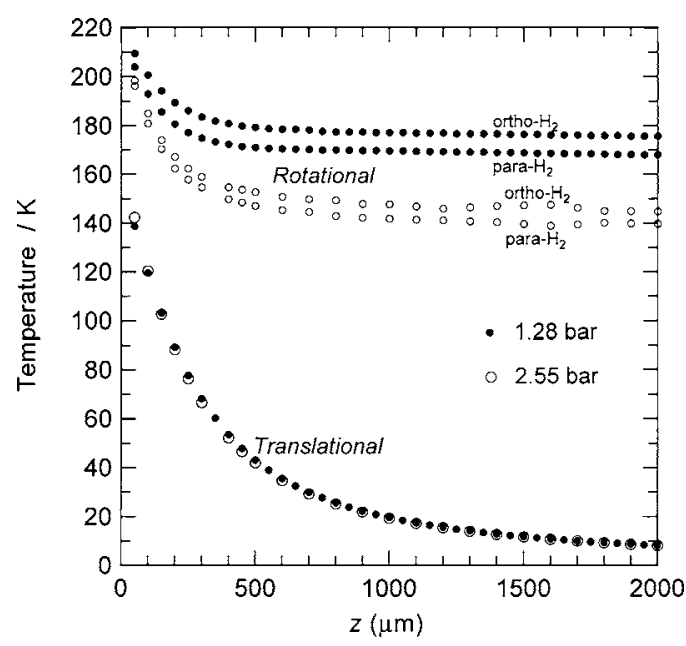

FIG. 3. Rotational and translational temperatures (smoothed data) along the axis of two supersonic expansions of $\mathrm{nH}_{2}$. Same conditions as in Fig. 1.

and $\mathrm{oH}_{2}$ along the $p_{0}=1.28$ bar expansion are shown in Fig. 4. The uncertainties in Fig. 4 are two-standard deviations from statistical errors plus an additional $10 \%$ for possible systematic errors not accounted for by the statistics.

\section{THEORETICAL CROSS SECTIONS AND RATES}

Comparing theory with experiment is a useful exercise for understanding in detail the elementary inelastic collisional processes in hydrogen. To this purpose a number of scattering calculations have been carried out within the framework of the quantum mechanical coupled channels method. ${ }^{5,19}$ In these calculations a rigid rotor potential energy surface (Diep and Johnson) ${ }^{20}$ has been employed on a grid of total energies from 354.35 to $5000 \mathrm{~cm}^{-1}$. At least two closed $(i, j)$ "well-ordered" rotational levels (i.e., with $i \leqslant j$ )

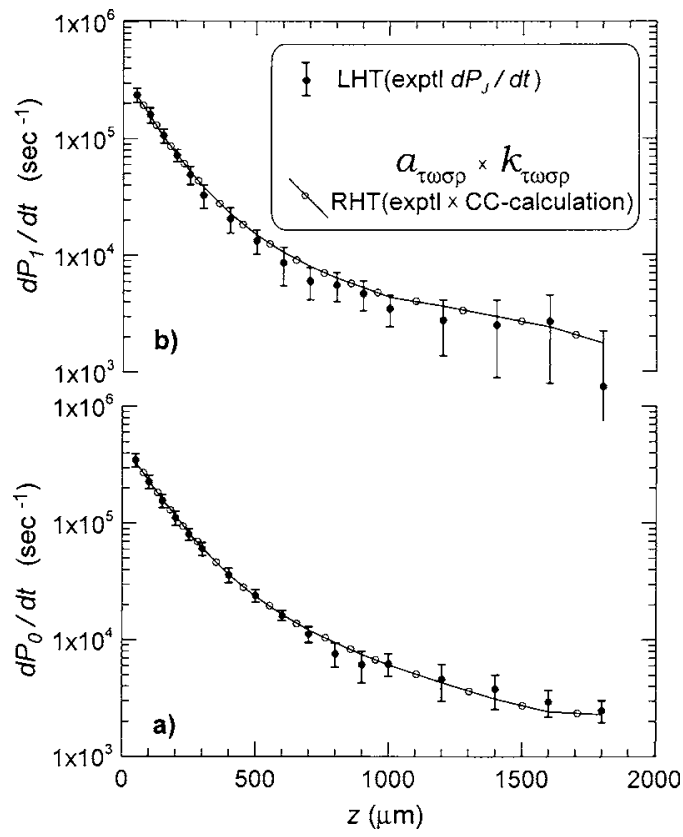

FIG. 4. Comparison left-hand term (LHT) vs right-hand term (RHT) of the master equation: (a) $J=0$ and (b) $J=1$ rotational population rates along the axis of the supersonic expansion of $\mathrm{nH}_{2}$ at 1.28 bars. Same conditions as in Fig. 1. The lines are a guide to the eye. 
TABLE I. Calculated $k_{\tau \omega \rightarrow \sigma \rho}^{\text {down }}$ rate coefficients for $\mathrm{H}_{2}: \mathrm{H}_{2}$ collisions (units of $10^{-20} \mathrm{~m}^{3} / \mathrm{s}$ ).

\begin{tabular}{|c|c|c|c|c|c|c|c|c|c|c|c|c|c|}
\hline $\begin{array}{r}\tau \omega \sigma \rho \\
\left|\Delta E / k_{B}\right| \longrightarrow_{t}(\mathrm{~K})\end{array}$ & $\begin{array}{l}3012 \\
4 \beta B\end{array}$ & $\begin{array}{l}4123 \\
4 \beta B\end{array}$ & $\begin{array}{l}2000 \\
6 \beta B\end{array}$ & $\begin{array}{l}2101 \\
6 \beta B\end{array}$ & $\begin{array}{c}2202 \\
6 \beta B\end{array}$ & $\begin{array}{c}2303 \\
6 \beta B\end{array}$ & $\begin{array}{c}0422 \\
8 \beta B\end{array}$ & $\begin{array}{c}3010 \\
10 \beta B\end{array}$ & $\begin{array}{c}3111 \\
10 \beta B\end{array}$ & $\begin{array}{c}3212 \\
10 \beta B\end{array}$ & $\begin{array}{c}3313 \\
10 \beta B\end{array}$ & $\begin{array}{c}2200 \\
12 \beta B\end{array}$ & $\begin{array}{c}2301 \\
16 \beta B\end{array}$ \\
\hline 300 & 864 & 631 & 167 & 283 & 281 & 291 & 258 & 135 & 164 & 164 & 177 & 4 & 5 \\
\hline 200 & 681 & 502 & 112 & 189 & 190 & 195 & 164 & 83 & 96 & 97 & 105 & 2 & 3 \\
\hline 150 & 574 & 428 & 86 & 145 & 146 & 151 & 122 & 61 & 68 & 70 & 75 & 1 & 2 \\
\hline 125 & 514 & 389 & 74 & 124 & 125 & 129 & 103 & 51 & 56 & 58 & 62 & 1 & 1 \\
\hline 100 & 452 & 350 & 63 & 104 & 106 & 109 & 86 & 42 & 45 & 47 & 51 & 1 & 1 \\
\hline 90 & 428 & 333 & 58 & 96 & 98 & 102 & 81 & 39 & 41 & 43 & 47 & 1 & 1 \\
\hline 80 & 403 & 319 & 54 & 89 & 91 & 94 & 75 & 36 & 37 & 39 & 43 & 1 & 1 \\
\hline 70 & 379 & 303 & 50 & 82 & 84 & 88 & 69 & 33 & 34 & 36 & 39 & 1 & 1 \\
\hline 60 & 356 & 292 & 47 & 76 & 78 & 81 & 64 & 30 & 31 & 33 & 36 & 1 & 1 \\
\hline 50 & 334 & 281 & 43 & 70 & 73 & 76 & 61 & 28 & 28 & 31 & 33 & 1 & 1 \\
\hline 40 & 319 & 279 & 40 & 65 & 68 & 71 & 61 & 27 & 26 & 29 & 32 & 0 & 1 \\
\hline 30 & 311 & 290 & 38 & 61 & 64 & 65 & 67 & 26 & 25 & 28 & 31 & 0 & 1 \\
\hline 20 & 330 & 359 & 39 & 60 & 64 & 60 & 86 & 28 & 27 & 27 & 33 & 0 & 1 \\
\hline 10 & 469 & 761 & 46 & 67 & 73 & 69 & 246 & 41 & 39 & 41 & 49 & 1 & 1 \\
\hline 5 & 979 & 3601 & 63 & 83 & 94 & 92 & 2035 & 89 & 83 & 95 & 107 & 1 & 2 \\
\hline
\end{tabular}

${ }^{\mathrm{a}} \beta B=84.98 \mathrm{~K}$.

were taken into account in addition to the open well-ordered ones. $^{3,5}$ The rotational energies were taken from Ref. 28.

For consistency between MEQ (1) and theory, the MOLSCAT code ${ }^{19}$ was employed considering indistinguishable molecules in $\mathrm{pH}_{2}: \mathrm{pH}_{2}$ and $\mathrm{oH}_{2}: \mathrm{oH}_{2}$ collisions. The cross sections for indistinguishable colliding molecules are expressed as ${ }^{3,5}$

$$
\sigma_{i j \rightarrow \ell m}^{\text {ind }}=W^{+} \sigma_{i j \rightarrow \ell m}^{+}+W^{-} \sigma_{i j \rightarrow \ell m}^{-},
$$

where $W^{+}=(I+1) /(2 I+1)$ and $W^{-}=I /(2 I+1)$ are the boson statistical weights of the symmetric and antisymmetric wave functions of the $\mathrm{H}_{2}-\mathrm{H}_{2}$ system due to the nuclear spin $\left(I=0\right.$ for $\mathrm{pH}_{2}, I=1$ for $\mathrm{oH}_{2}$ ) and

$$
\sigma_{i j \rightarrow \ell m}^{ \pm}=\left(\sigma_{i j \rightarrow \ell m}^{d}+\sigma_{i j \rightarrow m \ell}^{e}\right) \pm \sigma_{i j ; \ell m}^{d e} .
$$

$\sigma^{d}$ and $\sigma^{e}$ are the direct and the exchange cross sections for distinguishable molecules, while $\sigma^{d e}$ represents quantum interference effects, which have been shown to be negligible in the $\sigma_{00 \rightarrow 02}^{\text {ind }}$ cross section of $\mathrm{pH}_{2}{ }^{18}$ The $\sigma_{i j \rightarrow \ell m}^{\text {ind }}$ cross sections were obtained according to Takayanagi's counting of states. $^{21,29}$ By means of Eq. (12) the cross sections may also be expressed as

$$
\sigma_{i j \rightarrow \ell m}^{\text {ind }}=\sigma_{i j \rightarrow \ell m}^{d}+\sigma_{i j \rightarrow m \ell}^{e}+\left(W^{+}-W^{-}\right) \sigma_{i j ; \ell m}^{d e} .
$$

The state-to-state rate coefficients $k_{i j \rightarrow \ell m}$ at a kinetic temperature $T_{t}$ have been derived from the corresponding cross sections at energy $E$ by means of the weighted average

$$
k_{i j \rightarrow \ell m}\left(T_{t}\right)=\frac{\langle v\rangle}{\left(k_{B} T_{t}\right)^{2}} \int_{E_{S}}^{\infty} \frac{\sigma_{i j \rightarrow \ell m}(E)}{\exp \left(E / k_{B} T_{t}\right)} E d E,
$$

assuming that the colliding molecules obey a Maxwellian distribution at the temperature $T_{t} ; E=E_{\text {total }}-E_{i}-E_{j}$ is the available precollisional kinetic energy for the $J=i$ and $j$ rotational levels, $E_{s}$ is the minimum kinetic energy for the levels $J=\ell$ and $m$ to become accessible, and $\langle v\rangle$ $=\left(8 k_{B} T_{t} / \pi \mu\right)^{1 / 2}$ is the mean relative velocity of the colliding partners of reduced mass $\mu$.
The relevant down rate coefficients of $\mathrm{H}_{2}$ calculated from the PES of Diep and Johnson ${ }^{20}$ are shown in Table I, where $\beta=h c / k_{B}=1.4388 \mathrm{~K} / \mathrm{cm}^{-1}$ and $B$ is the rotational constant of $\mathrm{H}_{2}$. The homologous rates reported by Monchick and Schaefer $^{2}$ for $60 \leqslant T_{l} \leqslant 150 \mathrm{~K}$ are within $10 \%$ of those reported in Table I, while several rates at 100 and $300 \mathrm{~K}$ calculated by Le Bourlot et al. ${ }^{25}$ using Schwenke's PES (Ref. 30) agree within $25 \%$.

As can be seen in Table $\mathrm{I}$, from 5 to $300 \mathrm{~K}$ the $k^{\text {down }}$ rates only vary by one order of magnitude, facilitating an overview of the different contributions to the collisional energy transfer for various thermal conditions such as, for instance, along supersonic expansions.

\section{DISCUSSION}

The experimental and the theoretical results described above will be compared below according to two different approaches: the direct problem and the inverse problem.

In the direct problem a set of preexisting rate coefficients obtained, for instance, from theoretical calculations, or from an hypothetical independent experiment, will be assessed by means of the spectroscopic results from a supersonic expansion, according to the methodology of Sec. II.

In the inverse problem we will discuss how to obtain information about the rate coefficients from the supersonic expansion with as little aid as possible from preexisting theoretical values. In the ideal case of a perfect set of rate coefficients and an error-free experiment, the quantitative conclusions of the direct and inverse problems should be identical. In a real case, however, the various sources of uncertainty in the experiment and in the theoretical calculation impair such an ideal convergence. Nonetheless, much knowledge can be gained about the elementary collisional processes by comparing the conclusions from the direct and 
TABLE II. Statistics of the experiment.

\begin{tabular}{lcc}
\hline \hline Range $(\mathrm{K})$ & LHT/RHT $^{\mathrm{a}}$ & $N_{\text {data }}$ \\
\hline $90<T_{t} \leqslant 140$ & $1.03(6)$ & 16 \\
$40<T_{t} \leqslant 90$ & $1.06(14)$ & 16 \\
$20<T_{t} \leqslant 40$ & $0.99(20)$ & 16 \\
$10<T_{t} \leqslant 20$ & $1.02(20)$ & 18 \\
\hline \hline
\end{tabular}

${ }^{\mathrm{a}}$ One standard deviation in parentheses.

inverse problems. This is particularly relevant considering how difficult it is to retrieve significant information about the individual state-to-state rates for $\mathrm{H}_{2}: \mathrm{H}_{2}$ collisions from the experiments reported ${ }^{10,11}$ so far.

\section{A. The direct problem}

The rate coefficients of $\mathrm{H}_{2}$ calculated in Sec. IV are assessed in the range of kinetic temperatures $10 \leqslant T_{t} \leqslant 140 \mathrm{~K}$ by means of the two supersonic expansions of $\mathrm{nH}_{2}$ described in Sec. III. The rate coefficients $k_{\tau \omega \rightarrow \sigma \rho}^{\text {down }}$ to be tested are summarized in Table I, while the corresponding $a_{\tau \omega \sigma \rho}$ 's are given in the Appendix in terms of the experimental quantities $n, P_{i}$, and $T_{t}$ along the expansions.

The population rates $d P_{0} / d t$ and $d P_{1} / d t$ measured at the corresponding points of the expansions yield the left-hand term (LHT) of the MEQ (6). On the other hand, the sum of products $a_{\tau \omega \sigma \rho} k_{\tau \omega \rightarrow \sigma \rho}$ in Eqs. (A3) and (A4) of the Appendix yields the right-hand term (RHT) of the MEQ (6).

Comparing the experimental LHTs with the theoreticalexperimental RHTs for the $J=0$ and $J=1$ rotational levels of 1.28 and 2.55 bar expansions provides a consistency test of the calculated rates with the experiment. This comparison is shown in Fig. 4 for the 1.28 bar expansion. Similar results are obtained for the 2.55 bar expansion.

A summary of the statistics of the experiment is given in Table II. There the ratio LHT/RHT is employed as a figure of merit, whose ideal value should be LHT/RHT $=1 ; N_{\text {data }}$ are the number of experimental data points from both expansions, scrambling information about the $J=0$ and $J=1$ rotational levels in the referred thermal range.

The statistics of Table II suggests that the global accuracy of the $k_{\tau \omega \rightarrow \sigma \rho}^{\text {down }}$ rates of Table I is likely to be better than $10 \%$ for $90<T_{t} \leqslant 140 \mathrm{~K}$, better than $20 \%$ for $40<T_{t}$ $\leqslant 90 \mathrm{~K}$ and better than $25 \%$ for $10<T_{t} \leqslant 40 \mathrm{~K}$. Since the uncertainties of the experimental population rates $d P_{i} / d t$, coefficients $a_{\tau \omega \sigma \rho}$, and calculated $k_{\tau \omega \rightarrow \sigma \rho}^{\text {down }}$ 's are highly uncorrelated, the empirical ratio $0.99 \leqslant \mathrm{LHT} / \mathrm{RHT} \leqslant 1.06$ from Table II for a set of $N_{\text {data }}=66$ data points also suggests that the systematic overall uncertainty of the experiment is probably less than $10 \%$.

No information about the relative weight and accuracy of the individual collisional contributions to the population rates can be inferred from Table II. However, such information can be deduced primarily from the numerical values of the $a_{\tau \omega \sigma \rho}$ coefficients defined in Eqs. (A5) (Appendix), in combination with Table I. This shows that $21 \rightleftharpoons 01$ and $30 \rightleftharpoons 12$ are the dominant collisional induced transitions contributing to $d P_{0} / d t$, with approximate relative weights of $60 \%$ and $40 \%$. The remaining collisions contribute just mar-
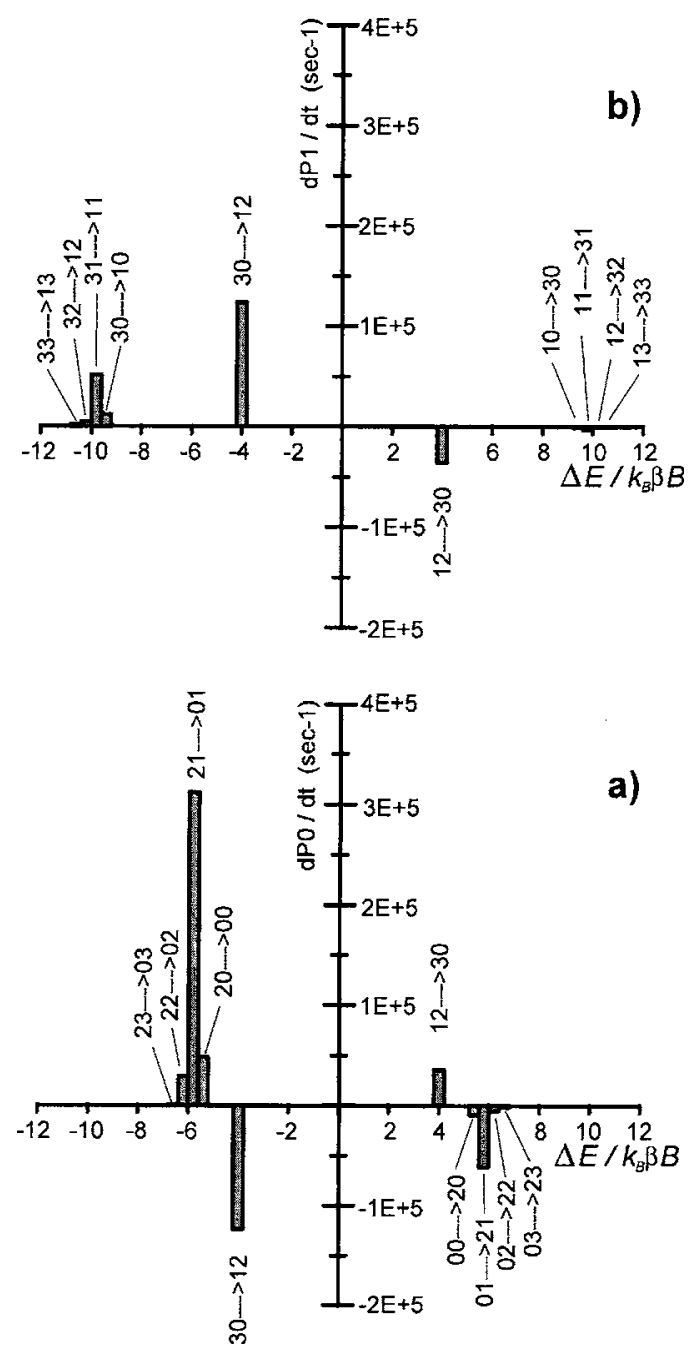

FIG. 5. Collisional spectrum of the $J=0$ (a) and $J=1$ (b) rotational population rates at the point $z=100 \mu \mathrm{m}$ of the supersonic expansion of $\mathrm{nH}_{2}$ at 1.28 bars. Same conditions as in Fig. 1.

ginally, their overall weight decreasing rapidly with the energy gap involved in the collision and with the precollisional rotational populations. For the $d P_{1} / d t$ rate, the dominant processes are $30 \rightleftharpoons 12$ and $31 \rightleftharpoons 11$, with relative weights of $70 \%$ and $30 \%$ and marginal contribution from other collisions. Thus the figure of merit LHT/RHT of Table II largely refers to the $21 \rightleftharpoons 01,30 \rightleftharpoons 12$, and $31 \rightleftharpoons 11$ contributions, in this order of importance. The corresponding rates on Table I are thus expected to be accurate to $\approx 10 \%$ for $90 \leqslant T_{t}$ $\leqslant 140 \mathrm{~K}, \approx 15 \%$ for $40 \leqslant T_{t} \leqslant 90 \mathrm{~K}$, and $\approx 20 \%$ for $10 \leqslant T_{t}$ $\leqslant 40 \mathrm{~K}$. Little can be said about the remaining rates, apart from their marginal importance in the thermal range of the experiment, $10 \leqslant T_{t} \leqslant 140 \mathrm{~K}$ and $140 \leqslant T_{r} \leqslant 210 \mathrm{~K}$.

The above information may be represented in terms of collisional spectra showing in detail the contribution of the various collisions to the population rates $d P_{0} / d t$ of $\mathrm{pH}_{2}$ and $d P_{1} / d t$ of $\mathrm{oH}_{2}$ at any point along the expansion of $\mathrm{nH}_{2}$. As an example the collisional spectra at a particular point of the 1.28 bar expansion are shown in Fig. 5.

In the collisional spectra the abscissa (energy) represents the global energy $\Delta E=k_{B} \beta B[\sigma(\sigma+1)+\rho(\rho+1)-\tau(\tau+1)$ $-\omega(\omega+1)]$ involved in the $\tau \omega \rightarrow \sigma \rho$ collisionally induced 
transition. Positive $\Delta E$ 's stand for globally up (or excitation) processes and negative $\Delta E$ 's stand for globally down (or deexcitation) processes. Note that some globally up or down processes are mixed "up-down" or "down-up" processes with simultaneous excitation of one molecule and deexcitation of the other. Symmetric $\pm \Delta E$ 's correspond to the timesymmetric $\sigma \rho \rightleftharpoons \tau \omega$ processes.

The ordinate (intensity) shows the contribution of a particular collision to the $J=0$ and $J=1$ rotational population rates. According to the scheme

$$
P_{J}(t+\Delta t)=P_{J}(t)+\frac{d P_{J}}{d t} \Delta t,
$$

positive intensities imply an increase of population in the rotational ground levels in time, i.e., an elementary cooling down process. Negative intensities are associated with a decrease of population of the ground levels, i.e., an elementary warming up process. Note that in each spectrum the sum of all positive and negative intensities yields a positive balance, accounting for the global rotational cooling of both species, $\mathrm{pH}_{2}$ and $\mathrm{oH}_{2}$, in the mixture of expanding $\mathrm{nH}_{2}$.

In the collisional spectra associated with the zone of silence of supersonic expansions the unbalance between positive and negative intensities increases at larger distances along the supersonic expansion and tends to vanish asymptotically at negative short distances, i.e., inside the nozzle, in the preexpansion chamber. At this point, positive and negative intensities become equal, the spectra showing inversion symmetry with respect to the origin. This reflects the thermodynamical equilibrium at the stagnation temperature and density, with a pictorial representation of the collisional status in detail.

The corresponding spectra at the same point of the 2.55 bar expansion are qualitatively similar to but somewhat more intense than those of the 1.28 bar expansion shown in Fig. 5. In general the global intensity of the collisional spectra decreases with distance $z$ by about the same factor of Fig. 4 , accompanied by moderate variations in the relative intensities associated with the various collisional transitions. This smooth trend may be expected to show a dramatic change at the normal shock wave associated with the end of the zone of silence of the expansions, where the flowing gas becomes suddenly rethermalized.

The spectra prove useful to represent graphically the collisional evolution of any nonequilibrium state regardless of how complex it may be, as for instance, within a shock wave. Note that the spectra are not universal, even under thermodynamical equilibrium, since they depend on the particular colliding species through the state-to-state rates, which are specific to the intermolecular potential.

\section{1. para- $\mathrm{H}_{2}$ rotational cooling in expanding $\mathrm{nH}_{2}$}

The spectrum in Fig. 5(a) shows that the dominant collisions contributing to the $d P_{0} / d t$ rate are the $21 \rightarrow 01$ ones, with a positive contribution. The time-symmetric collisions $01 \rightarrow 21$ have a far smaller negative contribution. The net balance is positive, implying that the net effect of the
$21 \rightleftharpoons 01$ collisions strongly increases the population rate of the ground level along the expansion, i.e., the efficiency of the rotational cooling of $\mathrm{pH}_{2}$.

It is somewhat surprising that the next most important contribution to $d P_{0} / d t$ is due to the $30 \rightarrow 12$ and $12 \rightarrow 30$ collisions, with a net negative contribution amounting to $\approx-0.25$ to -0.50 of the main one described above. Since all $k_{\tau \omega \rightarrow \sigma \rho}$ 's are positive by definition, this happens because the coefficient $a_{3012}$ is negative. Thus the $30 \rightarrow 12$ and $12 \rightarrow 30$ collisions tend to produce a net rotational heating of the $\mathrm{pH}_{2}$ fraction of expanding $\mathrm{nH}_{2}$. Such a peculiar behavior is to be expected for up-down or down-up collisions where one of the colliding partners becomes excited and the other one becomes simultaneously deexcited by a different amount of energy.

The $20 \rightarrow 00$ collisions in $\mathrm{nH}_{2}$ contribute to the cooling rate with only $15 \%-20 \%$ of the main contribution $21 \rightarrow 01$, while $22 \rightarrow 02$ with only $6 \%-10 \%$ and $23 \rightarrow 03$ with less than $4 \%$ of the main contribution, as shown in Fig. 5(a).

Note that the reversal collisions, at symmetric negative values of the energy gap $\Delta E$, also reverse the cooling down, or the heating up effects, but attenuated, reflecting the intrinsic nonequilibrium character of the supersonic expansion. The remaining collisions contributing to $d P_{0} / d t$ [see Eq. (A3) (Appendix)] only have a marginal effect on changes in the $J=0$ rotational populations of expanding $\mathrm{nH}_{2}$.

\section{2. ortho- $\mathrm{H}_{2}$ rotational cooling in expanding $\mathrm{nH}_{2}$}

As shown in Fig. 5(b) the main contributions to $d P_{1} / d t$ are positive. The dominant ones are the down-up $30 \rightarrow 12$ and the up-down $12 \rightarrow 30$ collisions. In contrast to $\mathrm{pH}_{2}$, the net contribution of these collisions in $\mathrm{oH}_{2}$ is the rotational cooling of the $\mathrm{oH}_{2}$ fraction of expanding $\mathrm{nH}_{2}$. The next contribution, amounting to about $50 \%$ of the former, arises from the down $31 \rightarrow 11$ and the up $11 \rightarrow 31$ collisions. The down $30 \rightarrow 10$ and the up $10 \rightarrow 30$ collisions only contribute with $\approx 10 \%$ to $\approx 15 \%$ of the dominant ones, while the remaining collisions have a marginal contribution in the investigated thermal range. The down-up $41 \rightarrow 23$ and the up-down $23 \rightarrow 41$ collisions have a marginal contribution in spite of the large $k_{41 \rightarrow 23}$ rate and the small total energy gap of $4 \beta B$ (see Table I) involved in these collisions, which are compensated by the very small value of the accompanying $a_{4123}$ coefficient.

On the whole, in a sample of expanding $\mathrm{nH}_{2}$ the global $d P_{0} / d t$ rate is positive and somewhat larger than the $d P_{1} / d t$ rate, as shown in Figs. 4 and 5. This implies that the rotational cooling efficiency of $\mathrm{pH}_{2}$ in $\mathrm{nH}_{2}$ is higher than that of $\mathrm{oH}_{2}$, leading to the $T_{r}\left(\mathrm{pH}_{2}\right)<T_{r}\left(\mathrm{oH}_{2}\right)$ breakdown of rotational temperatures shown in Fig. 3.

It may be concluded from this analysis that some down-up and up-down collisions are as relevant as or even more relevant for the rotational transfer of energy than the single down and up collisions involving a passive molecule. This effect is expected to be increasingly important at higher temperatures. 


\section{B. The inverse problem}

Equations (A3) and (A4) of the Appendix tell us that at a point $z$ of a particular $\mathrm{nH}_{2}$ expansion characterized by $n(z)$, $P_{i}(z)$, and $T_{t}(z)$, the number of unknowns $k_{\tau \omega \rightarrow \sigma \rho}$ is by far larger than the just two independent equations available, for $d P_{0} / d t$ and $d P_{1} / d t$. This a priori unfavorable situation can, in principle, be overcome by juxtaposing data from other expansions at different stagnation conditions, up to completing a linear system with more equations than $k^{\text {down }}$ unknowns. This strategy is impaired in practice, however, due to the strong correlation between homologous equations from different expansions and by the uncertainties of the experimental $d P_{i} / d t$ 's and $a_{\tau \omega \sigma \rho}$ coefficients.

As inferred from the direct problem, Fig. 5 shows that only three families of collisions contribute significantly to $d P_{0} / d t$ and $d P_{1} / d t: 30 \rightleftharpoons 12,2 m \rightleftharpoons 0 m$, and $3 m \rightleftharpoons 1 m$, with $m=0,1,2,3$. Other contributions to the $d P_{i} / d t$ 's are negligible since the corresponding $k_{\tau \omega \rightarrow \sigma \rho}$ (given in Table I) and/or the acompanying $a_{\tau \omega \sigma \rho}$ coefficients are small.

At the present level of experimental accuracy the only way to retrieve meaningful information about the significant $k_{\tau \omega \rightarrow \sigma \rho}$ rate coefficients from the supersonic expansion experiment is to impose some qualitative constraints on the basis of the above given arguments. Two sorts of constraints will be considered here: (i) over the $k_{\tau \omega \rightarrow \sigma \rho}^{\text {down }}$ unknowns, taking into account the theoretical results of Table I, and (ii) over the $a_{\tau \omega \sigma \rho}$ coefficients, according with their experimental values.

On this basis the following effective unknowns are proposed for $\mathrm{nH}_{2}: \mathrm{nH}_{2}$ collisions at $10 \leqslant T_{t} \leqslant 140 \mathrm{~K}$ and 140 $\leqslant T_{r} \leqslant 210 \mathrm{~K}$ :

$$
\begin{aligned}
& k_{30 \rightarrow 12}, \\
& k_{21 \rightarrow 01}=k_{22 \rightarrow 02}=k_{23 \rightarrow 03}=3 / 2 k_{20 \rightarrow 00}, \\
& k_{31 \rightarrow 11}=k_{32 \rightarrow 12}=k_{33 \rightarrow 13}=k_{30 \rightarrow 10},
\end{aligned}
$$

neglecting all other $a_{\tau \omega \sigma \rho} k_{\tau \omega \rightarrow \sigma \rho}^{\text {down }}$ terms.

In addition to the former constraints, one may observe from Table I that the $k_{\tau \omega \rightarrow \sigma \rho}^{\text {down }}$ 's are smooth functions of the translational temperature. This suggests partitioning the range of temperature $10 \leqslant T_{t} \leqslant 140 \mathrm{~K}$ into several subranges, augmenting the number of equations. Each subrange is then characterized by an average value of the three representative unknowns $\left\langle k_{21 \rightarrow 01}\right\rangle,\left\langle k_{30 \rightarrow 12}\right\rangle$, and $\left\langle k_{31 \rightarrow 11}\right\rangle$, with one equation per measured temperature point within the thermal subrange. The analysis of the system for each thermal subrange shows the strong correlation between $k_{30 \rightarrow 12}$ and $k_{31 \rightarrow 11}$, which cannot be broken down due to the relatively high experimental uncertainty. Only the combination $k_{30 \rightarrow 12}+b k_{31 \rightarrow 11}$, with $b$ $\approx 5$, is well defined under the present experimental conditions, the most relevant of which is the 3:1 fixed ratio of $\mathrm{oH}_{2}$ to $\mathrm{pH}_{2}$ nuclear spin variants in $\mathrm{nH}_{2}$.

The mean-square solution of the corresponding overdetermined systems for the four thermal subranges covering from $T_{t}=10$ up to $140 \mathrm{~K}$ is shown in Table III. The quoted experimental uncertainties include an estimate of the experimental error as well as the variation of $\left\langle k_{21 \rightarrow 01}\right\rangle$ or $\left\langle k_{30 \rightarrow 12}\right\rangle$
TABLE III. Experimental and theoretical state-to-state rates of $\mathrm{H}_{2}$ (units of $10^{-20} \mathrm{~m}^{3} \mathrm{~s}^{-1}$ ).

\begin{tabular}{ccc}
\hline \hline Subrange $(\mathrm{K})$ & $\left\langle k_{21 \rightarrow 01}\right\rangle$ & $\left\langle k_{30 \rightarrow 12}\right\rangle+5\left\langle k_{31 \rightarrow 11}\right\rangle$ \\
\hline \multirow{2}{*}{$90<T_{t} \leqslant 140$} & $115 \pm 31^{\mathrm{a}}$ & $776 \pm 159^{\mathrm{a}}$ \\
& $117 \pm 22^{\mathrm{b}}$ & $750 \pm 114^{\mathrm{b}}$ \\
& $133 \pm 24^{\mathrm{c}}$ & $703 \pm 129^{\mathrm{c}}$ \\
$40<T_{t} \leqslant 90$ & $75 \pm 19^{\mathrm{a}}$ & $555 \pm 32^{\mathrm{a}}$ \\
& $81 \pm 15^{\mathrm{b}}$ & $544 \pm 86^{\mathrm{b}}$ \\
$20<T_{t} \leqslant 40$ & $97 \pm 13^{\mathrm{c}}$ & $513 \pm 63^{\mathrm{c}}$ \\
& $62 \pm 15^{\mathrm{a}}$ & $429 \pm 36^{\mathrm{a}}$ \\
$10<T_{t} \leqslant 20$ & $63 \pm 3^{\mathrm{b}}$ & $501 \pm 45^{\mathrm{b}}$ \\
& $59 \pm 15^{\mathrm{a}}$ & $429 \pm 49^{\mathrm{a}}$ \\
& $64 \pm 5^{\mathrm{b}}$ & $613 \pm 150^{\mathrm{b}}$ \\
\hline \hline
\end{tabular}

${ }^{a}$ Experimental, this work.

${ }^{\mathrm{b}}$ Theory, this work.

${ }^{\mathrm{c}}$ Theory, adapted from Ref. 2.

$+5\left\langle k_{31 \rightarrow 11}\right\rangle$ within the corresponding thermal subrange; the uncertainty of the theoretical values only considers the latter variation. The results of Table III, although limited in practical terms, confirm again the good agreement of theory with experiment for the dominant rates $k_{21 \rightarrow 01}, k_{30 \rightarrow 12}$, and $k_{31 \rightarrow 11}$.

\section{Comparison with other experiments}

Due to the contribution from resonant rotation-rotation, rotation-translation, and elastic vibrational dephasing processes, the interpretation of line broadening coefficients of Raman lines of $\mathrm{H}_{2}$ in terms of state-to-state rate coefficients rests upon models. ${ }^{11}$ Therefore a comparison with present experiments will not be attempted here. On the other hand, relaxation times from ultrasonic absorption/dispersion experiments provide a simpler material to compare with. For two-level systems such as $\mathrm{oH}_{2}$ and $\mathrm{pH}_{2}$ at temperature $T_{t}$ $\leqslant 300 \mathrm{~K}$, the relaxation time may be expressed as ${ }^{1}$

$$
(p \tau)_{T_{t}}=\frac{1.363 \times 10^{-28} T_{t}}{k_{i \rightarrow f}+k_{f \rightarrow i}},
$$

where $(p \tau)$ is in atms for $T_{t}$ in Kelvin and $k$ 's in $10^{-20} \mathrm{~m}^{3} \mathrm{~s}^{-1} ; k_{i \rightleftharpoons f}$ refer to the $20 \rightleftharpoons 00$ rates of $\mathrm{pH}_{2}: \mathrm{pH}_{2}$ collisions, $31 \rightleftharpoons 11$ rates of $\mathrm{oH}_{2}: \mathrm{oH}_{2}$ collisions, and $21 \rightleftharpoons 01$ rates of $\mathrm{pH}_{2}: \mathrm{oH}_{2}$ collisions interchanging energy between the rotational levels of the vibrational ground state. The relaxation times calculated from the rates of Table I are shown in Table IV jointly with experimental results from ultrasonic measurements. This comparison confirms the good quality of the PES (Ref. 20) employed in the present close-coupling calculation. Tables III and IV also confirm indirectly the consistency of present experiment with the ultrasonic relaxation time measurements.

\section{SUMMARY AND CONCLUSIONS}

Some aspects of the complexity of the collisional problem of $\mathrm{H}_{2}$ are shown in this work. The experimental methodology employed is based on a fundamental kinetic equation, the so called "master equation" [Eq. (1)], which appears to be free from interpretative ambiguities for binary collisions. In it the state-to-state rate coefficients appear explicitly 
TABLE IV. Calculated and experimental relaxation times in $\mathrm{H}_{2}$ collisions $(p \tau)$ in units of $10^{-10}$ atm s.

\begin{tabular}{cccc}
\hline \hline$T_{t}(\mathrm{~K})$ & Transitions & $\begin{array}{c}\text { Expt. } \\
(p \tau)\end{array}$ & $\begin{array}{c}\text { Calc. }^{\mathrm{a}} \\
(p \tau)\end{array}$ \\
\hline 300 & $20 \rightleftharpoons 00$ & $130^{\mathrm{b}}$ & 128 \\
170 & & $187 \pm 2^{\mathrm{c}}$ & 192 \\
111.5 & & $223 \pm 4^{\mathrm{c}}$ & 212 \\
90.5 & & $211 \pm 2^{\mathrm{c}}$ & 207 \\
77 & & $220 \pm 8^{\mathrm{c}}$ & 197 \\
300 & $31 \rightleftharpoons 11$ & $226^{\mathrm{b}, \mathrm{d}}$ & $231^{\mathrm{e}}$ \\
77 & $30 \rightleftharpoons 10$ & $121 \pm 5^{\mathrm{c}}$ & 120 \\
\hline
\end{tabular}

${ }^{\mathrm{a}}$ This work, from Table I.

${ }^{\mathrm{b}}$ Reference 15 .

${ }^{\mathrm{c}}$ Reference 13 .

${ }^{\mathrm{d}}$ Reference 14.

${ }^{\mathrm{e}}$ Weighted over $31 \rightleftharpoons 11$ and $30 \rightleftharpoons 10$ in $\mathrm{nH}_{2}$.

as four-index rates which are free from averages over rotational states. Each index refers to a clearly identified rotational quantum state of both colliding molecules before and after the collision. This allows for unambiguous comparison with the results from high level quantum calculations, as well as with other experiments.

The following conclusions can be emphasized.

(1) The supersonic expansion spectroscopic experiment spanning the range $10<T_{t}<140 \mathrm{~K}$ confirms to $\approx 10 \%$ the accuracy of the $k_{30 \rightarrow 12}, k_{21 \rightarrow 01}$, and $k_{31 \rightarrow 11}$ rate coefficients for $\mathrm{H}_{2}: \mathrm{H}_{2}$ collisions derived from closecoupled calculations based on the PES of Diep and Johnson. $^{20}$

(2) The rates $k_{20 \rightarrow 00}, k_{21 \rightarrow 01}, k_{30 \rightarrow 10}$, and $k_{31 \rightarrow 11}$ calculated here reproduce the relaxation time measurements from ultrasonic absorption experiments at $77<T_{t}<300 \mathrm{~K}$ (Refs. 13-15) to better than 5\%.

(3) Appropriate reorganization of the master equation in the form of Eq. (6) allows for a detailed pictorial representation of the collisional status of any medium of instantaneous number density $n(t)$, kinetic temperature $T_{t}(t)$, and rotational distribution of populations $P_{i}(t)$ in the form of spectral diagrams as in Fig. 5. They are useful for visualizing the intricate internal energy transfer patterns due to collisions, regardless of equilibrium or nonequilibrium conditions.

(4) It is shown by experiment and calculations that the $30 \rightleftharpoons 12$ double process, with active participation of both molecules, one getting deexcited and the other getting excited in the collision by a different amount of energy, is as important as the single $21 \rightleftharpoons 01$ and $31 \rightleftharpoons 11$ processes where one of the molecules remains passive.

(5) The net balance of $30 \rightleftharpoons 12$ collisions in expanding $\mathrm{nH}_{2}$ is a heating up of the $\mathrm{pH}_{2}$ fraction and a simultaneous cooling down of the $\mathrm{oH}_{2}$ fraction, at the same speed, as shown in Fig. 5. Paradoxically, this does not lead to a violation of the 3:1 abundance ratio of ortho-para species in $\mathrm{nH}_{2}$. This ratio is always maintained along the expansion, as imposed in the master equation through the constraints given in the Appendix [Eq. (A1)].

(6) A behavior different from $30 \rightleftharpoons 12$ occurs for the $21 \rightleftharpoons 01$ and $31 \rightleftharpoons 11$ processes. Their net balance cools down, respectively, the $\mathrm{pH}_{2}$ and $\mathrm{oH}_{2}$ fractions of supersonically expanding $\mathrm{nH}_{2}$.

(7) The population or depletion of the $J=0$ level in $\mathrm{nH}_{2}$ is mainly due to the $21 \rightleftharpoons 01$ collisions in competition with the $30 \rightleftharpoons 12$ ones. The role of the $20 \rightleftharpoons 00$ processes is secondary, as shown in Fig. 5(a).

Concerning the experimental method the following conclusions can be drawn.

(1) Raman intensity measurements in supersonic jets were proven suited for investigating the rotation-translation energy transfer by collisions in a thermal range hardly accesible to any other experimental method. Jets provide collisions under controlled nonequilibrium conditions, depending on stagnation conditions, nozzle, and position, while Raman spectroscopy allows for unprecedented high spatial resolution and accuracy at any point of the jet. The wide range of temperatures, 10 $\leqslant T_{t} \leqslant 140 \mathrm{~K}$, has been investigated here in a single experiment.

(2) Nonetheless, the accuracy of the experimental results is still far from optimization. Translational temperatures in the experiment rest upon the hypothesis that the $\mathrm{H}_{2}$ jets are isentropic. Nonisentropic behavior might bias the determined rates to an extent that is not possible to quantify at the present state of the research. The fact that the kinetic temperatures of two different expansions are nearly identical is interpreted as a proof in favor of isentropic behavior.

(3) The main source of uncertainty in the upper end of the investigated thermal range, close to $T_{t}=140 \mathrm{~K}$ in this case, arises from the difficulty in determining accurately the position of the sonic surface of the expansion $(\mathrm{Mach}=1)$, apparently located inside the nozzle by about 100-200 $\mu \mathrm{m}$. This might cause some uncertainty in the initial flow velocity and in the population rates $d P_{i} / d t$ at the beginning of the expansion.

(4) The correlation between $k_{30 \rightarrow 12}$ and $k_{31 \rightarrow 11}$ could be broken down experimentally by expanding prepared $\mathrm{H}_{2}$ samples with different $\mathrm{oH}_{2}: \mathrm{pH}_{2}$ ratios. This can be inferred from Eqs. (A5) where the relative values of the $a_{3012}$ and $a_{3111}$ coefficients are shown to depend on the $\mathrm{oH}_{2}: \mathrm{pH}_{2}$ ratio through the $P_{1}, P_{3}, P_{0}$, and $P_{2}$ rotational populations.

(5) Encouragingly, the present experimental rates of $\mathrm{H}_{2}$, the close-coupling calculations, and the ultrasonic relaxation time measurements are in good agreement, in spite of the former limitations and uncertainties

Much experimental work remains to be done in order to improve our knowledge about $\mathrm{H}_{2}: \mathrm{H}_{2}$ collisions, in particular, to narrow the margin of uncertainty, to widen the thermal range, and to break the correlations between some observable rates by expanding $\mathrm{oH}_{2}: \mathrm{pH}_{2}$ mixtures of proportion different from the $3: 1$ in $\mathrm{nH}_{2}$. 


\section{ACKNOWLEDGMENTS}

This work has been supported by the Spanish Ministerio de Educación y Ciencia, Research Project No.FIS200402576. B Maté is acknowledged for her help in the initial stages of this work. Thanks are due to A. Simoni for the valuable comments on the paper.

\section{APPENDIX: MASTER EQUATION COEFFICIENTS}

In the thermal range investigated experimentally here, approximately $10 \leqslant T_{t} \leqslant 140 \mathrm{~K}$ and $140 \leqslant T_{r} \leqslant 210 \mathrm{~K}$, for the rotational levels of $\mathrm{H}_{2}$ of $J=0,1,2,3$, whose population can be measured with sufficient accuracy, the following constraints hold to a good approximation:

$$
\begin{aligned}
& P_{0}+P_{2}=1 / 4, \quad P_{1}+P_{3}=3 / 4, \\
& d P_{2} / d t=-d P_{0} / d t, \quad d P_{3} / d t=-d P_{1} / d t .
\end{aligned}
$$

In the referred thermal range the relevant terms of the master equation (6) are expressed as a function of the $k_{\tau \omega \rightarrow \sigma \rho}^{\text {down }}$ rates by

$$
\begin{aligned}
d P_{0} / d t= & a_{3012} k_{3012}+a_{2000} k_{2000}+a_{2101} k_{2101}+a_{2202} k_{2202} \\
& +a_{2303} k_{2303}+a_{0422} k_{0422}+a_{4103} k_{4103}+a_{2200} k_{2200} \\
& +a_{2301} k_{2301}, \\
d P_{1} / d t= & -a_{3012} k_{3012}+a_{4123} k_{4123}+a_{3010} k_{3010}+a_{3111} k_{3111} \\
& +a_{3212} k_{3212}+a_{3313} k_{3313}-a_{4103} k_{4103}+a_{2301} k_{2301},
\end{aligned}
$$

where the arrows are omitted for simplicity. The $a_{\tau \omega \sigma \rho}$ coefficients are given in the rigid rotor approximation by

$$
\begin{aligned}
& a_{3012}=n\left((7 / 15) P_{1} P_{2} e^{-4 \beta B / T_{t}}-P_{3} P_{0}\right), \\
& a_{4123}=n\left((27 / 35) P_{2} P_{3} e^{-4 \beta B / T_{t}}-P_{4} P_{1}\right), \\
& a_{2000}=n\left(-5 P_{0} P_{0} e^{-6 \beta B / T_{t}}+P_{2} P_{0}\right), \\
& a_{2101}=n\left(-5 P_{0} P_{1} e^{-6 \beta B / T_{t}}+P_{2} P_{1}\right), \\
& a_{2202}=n\left(-5 P_{0} P_{2} e^{-6 \beta B / T_{t}}+P_{2} P_{2}\right), \\
& a_{2303}=n\left(-5 P_{0} P_{3} e^{-6 \beta B / T_{t}}+P_{2} P_{3}\right), \\
& a_{0422}=n\left((9 / 25) P_{2} P_{2} e^{-8 \beta B / T_{t}}-P_{4} P_{0}\right), \\
& a_{3010}=n\left(-(7 / 3) P_{1} P_{0} e^{-10 \beta B / T_{t}}+P_{3} P_{0}\right),
\end{aligned}
$$

$$
\begin{aligned}
& a_{3111}=n\left(-(7 / 3) P_{1} P_{1} e^{-10 \beta B / T_{t}}+P_{3} P_{1}\right), \\
& a_{3212}=n\left(-(7 / 3) P_{1} P_{2} e^{-10 \beta B / T_{t}}+P_{3} P_{2}\right), \\
& a_{3313}=n\left(-(7 / 3) P_{1} P_{3} e^{-10 \beta B / T_{t}}+P_{3} P_{3}\right), \\
& a_{4103}=n\left(-(27 / 7) P_{0} P_{3} e^{-10 \beta B / T_{t}}+P_{4} P_{1}\right), \\
& a_{2200}=n\left(-50 P_{0} P_{0} e^{-12 \beta B / T_{t}}+2 P_{2} P_{2}\right), \\
& a_{2301}=n\left(-(35 / 3) P_{0} P_{1} e^{-16 \beta B / T_{t}}+P_{2} P_{3}\right) .
\end{aligned}
$$

${ }^{1}$ R. Ramaswamy, H. Rabitz, and S. Green, J. Chem. Phys. 66, 3021 (1977).

${ }^{2}$ L. Monchick and J. Schaefer, J. Chem. Phys. 73, 6153 (1980).

${ }^{3}$ G. Zarur and H. Rabitz, J. Chem. Phys. 60, 2057 (1974).

${ }^{4}$ H. Rabitz and S. Lam, J. Chem. Phys. 63, 3532 (1975).

${ }^{5}$ S. Green, J. Chem. Phys. 62, 2271 (1975).

${ }^{6}$ W. E. Köhler and J. Schaefer, J. Chem. Phys. 78, 6602 (1983).

${ }^{7}$ S. Y. Lin and H. Guo, J. Chem. Phys. 117, 5183 (2002).

${ }^{8}$ M. E. Mandy and S. K. Pogrebnya, J. Chem. Phys. 120, 5585 (2004).

${ }^{9}$ F. Gatti, F. Otto, S. Sukiasyan, and H. D. Meyer, J. Chem. Phys. 123, 174311 (2005).

${ }^{10}$ R. L. Farrow and D. W. Chandler, J. Chem. Phys. 89, 1994 (1988).

${ }^{11}$ L. A. Rahn, R. L. Farrow, and G. J. Rosasco, Phys. Rev. A 43, 6075 (1991).

${ }^{12}$ M. P. Le Flohic, P. Duggan, P. M. Sinclair, J. R. Drummond, and A. D. May, Can. J. Phys. 72, 186 (1994).

${ }^{13}$ R. M. Jonkman, G. J. Prangsma, I. Ertas, H. F. P. Knaap, and J. J. M. Beenakker, Physica (Amsterdam) 38, 441 (1968).

${ }^{14}$ L. M. Valley and R. C. Amme, J. Acoust. Soc. Am. 44, 1144 (1968).

${ }^{15}$ L. M. Valley and R. C. Amme, J. Chem. Phys. 50, 3190 (1969).

${ }^{16}$ J. E. Pollard, D. J. Trevor, Y. T. Lee, and D. A. Shirley, J. Chem. Phys. 77, 4818 (1982).

${ }^{17}$ M. J. Assael, S. Mixafendi, and W. A. Wakeman, J. Phys. Chem. Ref. Data 15, 1315 (1986).

${ }^{18}$ B. Maté, F. Thibault, G. Tejeda, J. M. Fernández, and S. Montero, J. Chem. Phys. 122, 064313 (2005).

${ }^{19}$ J. M. Hutson and S. Green, MOLSCAT, version 14, Collaborative Computational Project no 6 of the UK Science and Engineering Research Council, 1994.

${ }^{20}$ P. Diep and J. K. Johnson, J. Chem. Phys. 112, 4465 (2000); 113, 3480 (2000).

${ }^{21}$ G. Danby, D. R. Flower, and T. S. Monteiro, Mon. Not. R. Astron. Soc. 226, 739 (1987).

${ }^{22}$ D. R. Flower, Mon. Not. R. Astron. Soc. 297, 334 (1998).

${ }^{23}$ D. R. Flower and E. Roueff, J. Phys. B 31, 2935 (1998).

${ }^{24}$ D. R. Flower and E. Roueff, J. Phys. B 32, 3399 (1999).

${ }^{25}$ J. Le Bourlot, G. Pineau des Forets, and D. R. Flower, Mon. Not. R. Astron. Soc. 305, 802 (1999).

${ }^{26}$ D. R. Flower and G. Pineau des Forets, Mon. Not. R. Astron. Soc. 316, 901 (2000).

${ }^{27}$ A. Ramos, G. Tejeda, J. M. Fernández, and S. Montero, Phys. Rev. A 66, 022702 (2002).

${ }^{28}$ C. Schwartz and R. J. Le Roy, J. Mol. Spectrosc. 121, 420 (1987).

${ }^{29}$ K. Takayanagi, Adv. At. Mol. Phys. 1, 149 (1965).

${ }^{30}$ D. W. Schwenke, J. Chem. Phys. 89, 2076 (1988). 